\title{
Lista de crustáceos distribuidos en troncos hundidos en el humedal tropical Pantanos de Centla, al sur del golfo de México
}

\author{
List of crustaceans distributed in submerged woody debris in the tropical wetlands of \\ Pantanos de Centla, southern Gulf of Mexico
}

\author{
Hugo Montalvo-Urgel, Alberto J. Sánchez*, Rosa Florido y Alberto A. Macossay-Cortez \\ Laboratorio de Hidrobiología, Diagnóstico y Manejo de Humedales Tropicales, División Académica de Ciencias Biológicas, Universidad Juárez \\ Autónoma de Tabasco. Km. 0.5 carretera Villahermosa-Cárdenas, 86039 Villahermosa, Tabasco, México. \\ *Correspondencia: alberthoj.sanchez@gmail.com
}

\begin{abstract}
Resumen. En las comunidades acuáticas, la complejidad del hábitat modifica la dinámica y la estructura, ya que la diversidad y abundancia aumentan conforme se incrementan las estructuras físicas emergentes del sustrato, como los troncos hundidos, que son sitios de refugio, alimentación y reproducción de la fauna asociada a esos hábitats. En 18 lagunas de Pantanos de Centla, Tabasco, donde se encontraron troncos hundidos, se realizaron 2 muestros manuales en la temporada de máxima inundación y 1 en la de mínima inundación. Se capturaron 1228 crustáceos que pertenecen a 4 órdenes, 11 familias, 11 géneros y 13 especies, de los cuales los malacostracos predominaron con 12 especies. Las 13 especies de crustáceos registradas en este trabajo representaron el $17 \%$ de la carcinofauna capturada en los ecosistemas limnéticos del estado de Tabasco. El anfípodo Hyalella azteca fue numéricamente la especie dominante. La distribución de Balanus improvisus, Uhlorchestia ulheri, Platychirograpsus spectabilis, Armases cinereum y Goniopsis cruentata estuvo restringida principalmente a la zona de influencia marina. Balanus improvisus y Sphaeroma terebrans fueron las únicas especies sésiles y taladradoras recolectadas. Para las restantes 11 especies de crustáceos, los troncos hundidos también son un hábitat alternativo.
\end{abstract}

Palabras clave: macrocrustáceos, troncos hundidos, humedal tropical, Pantanos de Centla.

\begin{abstract}
The dynamics and structure of aquatic communities are affected by the habitat complexity as diversity and abundance increase with the availability of structured habitats such as coarse woody debris that provides sites used for refuge, feeding and reproduction. Manual sampling of the fauna associated with this habitat was carried out twice during the season of maximum floods and once during minimum flooding, in 18 lagoons of Pantanos de Centla with coarse woody debris. The 1228 crustaceans collected belong to 4 orders, 11 families, 11 genera and 13 species, among which malacostracans dominated with 12 species. The 13 species of crustaceans reported here represented $17 \%$ of the total carcinofauna recorded in the freshwater ecosystems of Tabasco. The amphipod Hyalella azteca was the numerically dominant species. The distribution of Balanus improvisus, Uhlorchestia ulheri, Platychirograpsus spectabilis, Armases cinereum and Goniopsis cruentata was restricted mainly to the area of marine influence. Moreover, B. improvisus and Sphaeroma terebrans were the only 2 sessile and wood-boring species. Coarse woody debris is also an alternative habitat for the other 11 species.
\end{abstract}

Key words: macrocrustaceans, coarse woody debris, tropical wetland, Pantanos de Centla.

\section{Introducción}

La distribución y diversidad de la fauna registrada en los ecosistemas acuáticos epicontinentales habitualmente se explica en función de la geomorfología y la presencia de hábitats con estructuras físicas emergentes o estructurados (Pelicice y Agostinho, 2006; Rozas y Minello, 2006; Sammons y Maceina, 2006; Yamazaki et al., 2006).

Recibido: 19 enero 2010; aceptado: 26 abril 2010
En estos hábitats, las raíces de manglar y las diferentes asociaciones de macrófitas sumergidas enraizadas, emergentes enraizadas y libres flotadoras sobresalen por albergar mayor abundancia y diversidad (Heck y Crowder, 1991; Primavera, 1997; Rozas y Minello, 2006; Guevara et al., 2007; Rocha-Ramírez et al., 2007).

Los troncos hundidos, originados por la vegetación arbórea marginal, también representan un hábitat estructurado que sostiene alta abundancia y diversidad de fauna asociada (Everett y Ruiz, 1993; Sánchez et al., 
1996; Corona et al., 2000; Florido et al., 2000; Cheruvelil et al., 2002; Tietje et al., 2002). Los troncos hundidos funcionan como sitios de alimentación, protección contra los depredadores, reproducción y ovoposición (Thorp et al., 1985; Harmon et al., 1986; O’Connor, 1991; Fausch y Northcote, 1992). Adicionalmente, este hábitat es uno de los escasos sustratos duros que proporcionan espacios colonizables para organismos taladradores y sésiles (Sedell et al., 1982; Triska et al., 1982; Florido et al., 2000) y aumenta directa e indirectamente la disponibilidad de alimentos, ya sea por consumo directo de madera o por la depredación de los organismos adheridos o asociados a ésta (Cummins et al., 1983; Britton y Morton, 1988; Lockaby et al., 2002). Por último, los troncos hundidos propician la depositación de sedimentos y materia orgánica, y funcionan como almacén de energía y carbono (Triska et al., 1982; Harmon et al., 1986).

En Pantanos de Centla se han registrado 21 especies de crustáceos (Florido et al., 2000; Velasco et al., 2005; Cruz-Ascencio et al., 2009), de las cuales sólo Florido et al. (2000) incluyen registros provenientes de muestreos efectuados en troncos hundidos. Además, la información publicada sobre crustáceos distribuidos en troncos hundidos en otros ecosistemas limnéticos y estuarinos ubicados en la ecorregión del Usumacinta y en la planicie costera del golfo de México es limitada (Corona et al., 2000; Sánchez et al., 2007a), lo que constituye un vacío en el conocimiento sobre la distribución global de la fauna (Lévêque et al., 2005), que la lista de especies de crustáceos incluida en este estudio subsana parcialmente.

\section{Material y métodos}

La Reserva de la Biosfera Pantanos de Centla ocupa 302706 ha y está localizada en la cuenca baja de los ríos Usumacinta-Grijalva (1757'53” y 18³9'03" N 9206’39” y 9245’58” O) (Bautista-Jiménez et al., 2000). La descarga de los ríos Grijalva-Usumacinta es la tercera en importancia en el golfo de México, después de los ríos Mississippi y Atchafalaya (Velázquez-Villegas, 1994; Collier y Halliday, 2000).

Para obtener un muestreo representativo de la fauna asociada a los troncos hundidos en Pantanos de Centla se seleccionaron 19 ecosistemas lagunares permanentes (Cuadro 1) con base en la distribución de la vegetación arbórea, la distribución de los ecosistemas lénticos y lóticos y la accesibilidad a los sitios de muestreo.

Los troncos hundidos se muestrearon en 3 ocasiones; 2 en la temporada de máxima inundación (noviembre y diciembre, 2000 y 2001) y 1 en la de mínima inundación (mayo y junio, 2001), para cubrir los extremos del ciclo de inundación registrados en el área, ya que la dinámica y la organización de las comunidades acuáticas se

Cuadro 1. Ubicación de las localidades en Pantanos de Centla. Velázquez-Villegas (1994)

\begin{tabular}{|c|c|c|c|c|}
\hline Laguna & Posición geográfica (UTM) & Subcuenca & $T\left({ }^{\circ} \mathrm{C}\right)$ & $S$ (ups) \\
\hline Laguna San Pedrito & $542550-2030632$ & $\mathrm{U}$ & 27.8 & 0.92 \\
\hline Laguna Punteada & $561812-2030260$ & $\mathrm{U}$ & 28.6 & 0.61 \\
\hline Laguna El Guanal & $558711-2022995$ & $\mathrm{U}$ & 28.1 & 0.19 \\
\hline Laguna San Isidro & $555330-2035112$ & $\mathrm{U}$ & 29.1 & 0.42 \\
\hline Laguna El Coco & $532455-2043530$ & $\mathrm{~TB}$ & 29.5 & 0.69 \\
\hline Laguna El Cometa & $558134-2041912$ & $\mathrm{U}$ & 28.5 & 2.76 \\
\hline Laguna Narváez & $581337-2036132$ & $\mathrm{U}$ & 30.0 & 0.60 \\
\hline Laguna El Viento & $536135-2015450$ & $\mathrm{~TB}$ & 28.6 & 0.08 \\
\hline Laguna Concepción & $580726-2028665$ & $\mathrm{G}$ & 28.6 & 0.38 \\
\hline Laguna Larga & $541622-2012153$ & G & 29.0 & 0.33 \\
\hline Laguna Los Ídolos & $538880-2021224$ & $\mathrm{~TB}$ & 28.6 & 0.47 \\
\hline Laguna El Tintal & $541326-1999982$ & $\mathrm{G}$ & 26.8 & 0.06 \\
\hline Laguna Landeros & $559537-2002719$ & G & 30.0 & 0.33 \\
\hline Laguna Sargazal & $567113-2003620$ & G & 30.5 & 0.33 \\
\hline Laguna El Loncho & $565334-2001124$ & G & 30.3 & 0.16 \\
\hline Laguna Chichicastle & $561616-2013710$ & $\mathrm{U}$ & 28.5 & 0.16 \\
\hline Laguna El Sauzo* & $567332-2013974$ & $\mathrm{U}$ & 29.6 & 0.33 \\
\hline Laguna San Isidro El Jobo & $576193-2008687$ & $\mathrm{U}$ & 29.1 & 0.16 \\
\hline Laguna Cantemoc & $569920-2015809$ & $\mathrm{U}$ & 29.1 & 0.76 \\
\hline
\end{tabular}

$T=$ temperatura promedio anual, $S=$ salinidad promedio anual. .Subcuencas hidrológicas. $\mathrm{U}=\mathrm{Usumacinta}, \mathrm{G}=\mathrm{Grijalva}, \mathrm{TB}=\mathrm{Tres}$ Brazos, definidas por Velázquez-Villegas (1994).

$*=$ laguna $\sin$ troncos hundidos. 
encuentran reguladas por estos ciclos (Bacon, 1996). Los troncos hundidos se extrajeron del agua en forma manual y rápida para evitar el escape de organismos ( $\mathrm{O}^{\prime}$ Connor, 1991; Florido et al., 2000). La recolecta de organismos sobre la superficie del tronco y dentro de las galerías se realizó en campo y terminó cuando no se detectaron más organismos.

La fauna asociada se identificó con base en los caracteres taxonómicos propuestos por: Pilsbry (1916) para cirripedios, Bousfield (1973), Lincoln (1979), Barnard y Karaman (1991), González y Watling (2002), Myers y Lowry (2003) y Serejo (2004) para anfípodos, Schultz (1969) y Kensley y Schotte (1989) para isópodos, Holthuis (1952) y Nates y Villalobos-Hiriart (1990) para camarones carideos y Williams (1984) para cangrejos braquiuros. Para cada especie, la lista incluye nombre científico, distribución geográfica, registros en México, distribución local y observaciones. En la lista, las especies se ordenaron bajo el orden filogenético propuesto por Martin y Davis (2001).

Para las entradas Distribución y Registros en México se consideraron los datos citados en la literatura. La Distribución local incluye el material examinado, los datos morfológicos, los sitios donde cada especie fue recolectada en este estudio y el intervalo de la temperatura $\left({ }^{\circ} \mathrm{C}\right)$ y salinidad (ups) registrados durante los muestreos. El Material examinado incluye el número total de organismos recolectados y la longitud total (LT) promedio con su intervalo anotado entre paréntesis. Se anotaron los valores de densidad de los órdenes Amphipoda e Isopoda (ind/ $\mathrm{m}^{3}$ ), y los de Sessilia y Decapoda (ind $/ \mathrm{m}^{2}$ ), para mostrar tendencias cualitativas de su distribución local. Las
Observaciones se refirieren a la distribución de las especies con respecto a ecosistemas, hábitat y hábitos alimenticios.

\section{Resultados}

En 18 de las 19 lagunas muestreadas se encontraron troncos hundidos. La excepción fue la laguna El Sauzo, la cual se excluyó en los análisis de este estudio (Cuadro 1).

Los 1228 crustáceos capturados pertenecieron a 2 clases, 4 órdenes, 11 familias, 11 géneros y 13 especies. La clase Malacostraca predominó con el $93 \%$ del total de crustáceos, con 3 órdenes, 10 familias, 10 géneros y 12 especies (Cuadro 2).

\section{Lista de especies}

Phylum Arthropoda

Clase Maxillopoda Dahl, 1956

Infraclase Cirripedia Burmeister, 1834

Familia Balanidae Leach, 1817

Balanus improvisus Darwin, 1854

Distribución. Desde Escocia hasta la costa francesa en Europa. En América desde Nueva Escocia, Canadá, hasta la Patagonia, Argentina (Pilsbry, 1916; Lippson y Lippson, 1984; Britton y Morton, 1988).

Registro en México. En La Pesca, Tamaulipas y en la laguna San Pedrito en Pantanos de Centla, Tabasco (Florido et al., 2000; Celis, 2004; Álvarez et al., 2005).

Distribución local - 92 ejemplares. LT promedio $0.77 \mathrm{~cm}$ $(0.44$ a $9.04 \mathrm{~cm})$ en densidades entre 1.06 y $83.54 \mathrm{ind} / \mathrm{m}^{2}$. En las lagunas San Pedrito, El Cometa y El Coco entre 27.8 y $29^{\circ} \mathrm{C}$ y desde 0.69 hasta 2.76 ups.

Cuadro 2. Macrocrustáceos asociados con troncos hundidos en la Reserva de la Biosfera Pantanos de Centla

\begin{tabular}{|c|c|c|c|c|}
\hline Clase & Orden & Familia & Especie & Abundancia \\
\hline Maxillopoda & Sessilia & Balanidae & Balanus improvisus & 92 \\
\hline \multirow[t]{12}{*}{ Malacostraca } & Amphipoda & Hyalellidae & Hyalella azteca & 1035 \\
\hline & & Talitridae & Uhlorchestia ulheri & 2 \\
\hline & Isopoda & Sphaeromatidae & Sphaeroma terebrans & 39 \\
\hline & Decapoda & Atyidae & Potimirim mexicana & 22 \\
\hline & & Palaemonidae & Macrobrachium acanthurus & 2 \\
\hline & & & Macrobrachium hobbsi & 11 \\
\hline & & & Macrobrachium olfersii & 1 \\
\hline & & Cambaridae & Procambarus(Austrocambarus) llamasi & 7 \\
\hline & & Panopeidae & Rhithropanopeus harrisii & 11 \\
\hline & & Glyptograpsidae & Platychirograpsus spectabilis & 3 \\
\hline & & Sesarmidae & Armases cinereum & 1 \\
\hline & & Grapsidae & Goniopsis cruentata & 2 \\
\hline Total & & & & 1228 \\
\hline
\end{tabular}


Observaciones. En muelles y junto con Balanus eburneus (Gould, 1841) han representado las especies más comunes adheridas a pilotes, embarcaderos y otros sustratos duros, así como raíces de manglar, conchas, rocas, cascos de barcos y troncos hundidos (Pilsbry, 1916; Britton y Morton, 1988; Florido et al., 2000; Álvarez et al., 2005).

Clase Malacostraca Latreille, 1802

Orden Amphipoda Latreille, 1816

Familia Hyalellidae Bulycheva, 1957

Hyalella azteca Saussure, 1858

Distribución. Desde Maine, EUA hasta la Patagonia, Argentina y Hawaii, EUA (Saussure, 1858; González y Watling, 2001).

Registro en México. En el lago de Chapultepec, ciudad de México; cuenca Lerma-Chapala, Michoacán; lagos Alchichica, Atexcac, Quechulac, La Preciosa, Aljojuca y Tecuitlapa, Puebla; lagunas El Limón, Estancia Vieja, Bayasú, Chifladora, Julivá, Horizontes y La Mona, en el río González y ampliamente distribuida en Pantanos de Centla en Tabasco (Alcocer et al., 2000; González y Watling, 2002; Velasco, 2004; Álvarez et al., 2005; Barba y Sánchez, 2007).

Distribución local -1 035 ejemplares. LT promedio 0.20 $\mathrm{cm}(0.15-0.34 \mathrm{~cm})$ en densidades entre 10.12 y 2349.37 ind $/ \mathrm{m}^{3}$. En las lagunas San Pedrito, Punteada, El Guanal, San Isidro, El Cometa, Narváez, Chichicastle, San Isidro-El Jobo, Cantemoc, Concepción, Larga, El Tintal, Landeros, Sargazal, Loncho, El Coco, El Viento y Los Ídolos, entre 27.8 y $30{ }^{\circ} \mathrm{C}$ y de 0.16 a 2.76 ups.

Observaciones. Anfípodo asociado con las raíces del lirio acuático, vegetación acuática sumergida, vegetación marginal y ocasionalmente en sustratos suaves sin vegetación (Alcocer et al., 2000; Álvarez et al., 2005; Barba y Sánchez, 2007).

Familia Talitridae Stebbing, 1906

Uhlorchestia uhleri (Shoemaker, 1930)

Distribución. Desde Carolina del Norte hacia la costa este de Florida, este del delta del río Mississippi, este de Texas en EUA, hasta la cuenca del río Usumacinta-Grijalva (Bousfield y Heard, 1986; Velasco et al., 2005).

Registro en México. En río Playa en la cuenca del Coatzacoalcos y en las lagunas San Pedrito, El Cometa, El Coco y Chichicastle en Pantanos de Centla ubicado en la cuenca baja de los ríos Usumacinta-Grijalva, Tabasco (Velasco et al., 2005).

Distribución local - 2 ejemplares. LT 0.16 - $0.18 \mathrm{~cm}$, en densidades entre 15.35 a $274.92 \mathrm{ind} / \mathrm{m}^{3}$. En las lagunas $\mathrm{El}$ Coco y El Cometa entre 28.5 y $29.5^{\circ} \mathrm{C}$ y de 0.69 a 2.76 ups.

Observaciones. Especie distribuida en Spartina patens
(Ait.) Muhl., en asociaciones de vegetación enraizada emergente, macrófitas enraizadas sumergidas, en raíces de jacinto acuático, Eichhornia crassipes (Mart.) Solms., y mangle rojo (Rhizophora mangle L.), troncos hundidos $\mathrm{y}$ debajo de restos de troncos y detrito (Bousfield y Heard, 1986; Velasco et al., 2005).

Orden Isopoda Latreille, 1817

Familia Sphaeromatidae Latreille, 1825

Sphaeroma terebrans Bate, 1866

Distribución. Desde Virginia hasta Florida en la costa este de los EUA, Belice, Venezuela, Brasil y Cuba. En Nigeria, costa sureste de África y a lo largo de las costas de la India y Australia (Pennak, 1978; Britton y Morton, 1988; Kensley y Schotte, 1989; Ellison y Farnsworth, 1990).

Registro en México. En la laguna San Pedrito en Pantanos de Centla, Tabasco (Florido et al., 2000).

Distribución local - 39 ejemplares. LT promedio $0.71 \mathrm{~cm}$ (0.44 - $0.9 \mathrm{~cm})$ en densidades de 14.76 a $183.93 \mathrm{ind} / \mathrm{m}^{3}$. En las lagunas San Pedrito, El Guanal y El Coco entre 27.8 y $29.5^{\circ} \mathrm{C}$ y de 0.19 a 0.92 ups.

Observaciones. Especie horadadora y taladradora de raíces y tallos del mangle rojo $R$. mangle, en pilotes de concreto y madera, troncos hundidos y en conchas vacías de Balanus sp. (Pennak, 1978; Kensley y Schotte, 1989; Florido et al., 2000, Álvarez et al., 2005).

Orden Decapoda Latreille, 1802

Familia Atyidae de Haan, 1849

Potimirim mexicana (Saussure, 1857)

Distribución. Noreste de México hasta Panamá. En Cuba, Jamaica y Puerto Rico (Chace y Hobbs, 1969; Chace, 1972; Abele y Kim, 1989).

Registro en México. Estero de Canalá, Guayabitos en Nayarit en la costa del Pacífico mexicano. En la vertiente del golfo de México en la cuenca del río Soto la Marina y río Coy en San Luis Potosí; Parque Quevedo y Playa Norte en Veracruz; cuenca de los ríos Grijalva y Usumacinta, a lo largo de los ríos Mezcalapa-Carrizal, Samaria, Tonalá y González, en Pantanos de Centla en Tabasco; ríos Candelaria, Chumpán y Palizada que desembocan en la laguna de Términos, Campeche (Villalobos-Figueroa, 1959, 1982, 1983; Rodríguez-Almaraz y Campos, 1996; Suárez, 2002; Álvarez et al., 2005).

Distribución local - 22 ejemplares. LT promedio $0.27 \mathrm{~cm}$ $(0.11$ a $0.41 \mathrm{~cm})$ en densidades de 2.4 a $21.8 \mathrm{ind} / \mathrm{m}^{2}$. En las lagunas San Isidro y El Viento entre 28.6 y $29.1{ }^{\circ} \mathrm{C}$ y de 0.08 a 0.42 ups.

Observaciones. Distribuidos frecuentemente entre las hojas y buscan la protección mecánica que pueden brindarle las raíces de las plantas acuáticas o las raíces de las plantas que crecen en los márgenes de los ríos. Habitan 
desde pequeños arroyos de montaña con un mínimo de agua hasta grandes ríos en la llanura costera (VillalobosFigueroa, 1959; Álvarez et al., 2005).

Familia Palaemonidae Rafinesque, 1815

Macrobrachium acanthurus (Wiegmann, 1836)

Distribución. Desde el estuario Neuse River, Carolina del Norte y río Bravo en Texas, EUA, hasta río Grande do Sul, Brasil; Cuba, Haití, República Dominicana y Puerto Rico (Holthuis, 1952; Chace y Hobbs, 1969; Chace, 1972; Williams, 1984; Vargas y Cortés, 1999; Almeida et al., 2008).

Registro en México. Desde el río Bravo, Tamaulipas, río Ramos en la cuenca del San Juan, Nuevo León y en el río Huitzilapan, Veracruz. En los ríos González, laguna Mecoacán, Mezcalapa-Carrizal, Samaria, González, San Pedro y Puyacatengo, y laguna Yucateco, así como en Pantanos de Centla en Tabasco y en Quintana Roo (Granados, 1984; Rodríguez-Almaraz y Campos, 1996; Álvarez et al., 1999; Mejía-Ortiz et al., 2001, Suárez, 2002; Domínguez et al., 2003; Álvarez et al., 2005).

Distribución local - 2 ejemplares. LT $0.12-0.35 \mathrm{~cm}$, en densidades entre 0.88 a $1.03 \mathrm{ind} / \mathrm{m}^{2}$. En las lagunas $\mathrm{El}$ Viento y El Coco entre 28.6 y $29.5{ }^{\circ} \mathrm{C}$ y de 0.08 a 0.69 ups.

Observaciones. Distribuido en la zona costera en ríos y bahías, en fondos lodosos y zonas con poca corriente, cercanas a la orilla, donde busca el refugio de hábitats estructurados (Williams, 1984; Rodríguez, 1993).

Macrobrachium hobbsi (Nates y Villalobos, 1990)

Distribución. En ambas vertientes en la porción occidental de México (Villalobos-Hiriart et al., 1993; Florido et al., 2000, Álvarez et al., 2005).

Registro en México. En el Pacífico mexicano desde Pijijiapan, Nayarit hasta el río El Naranjo en Chiapas. En el golfo de México desde el río Coy, San Luís Potosí, ríos Mezcalapa-Carrizal, Samaria y Puyacatengo, laguna La Mona hasta laguna San Pedrito en Pantanos de Centla, Tabasco (Nates y Villalobos-Hiriart, 1990; VillalobosHiriart et al., 1993; Molinero, 1996; Rodríguez-Almaraz y Campos, 1996; Álvarez et al., 1999; Florido et al., 2000; Raz-Guzman y Hermoso, 2002; Suárez, 2002; Corona y Raz-Guzman, 2003; Álvarez et al., 2005).

Distribución local -11 ejemplares. LT promedio $0.94 \mathrm{~cm}$ $(0.42-1.98 \mathrm{~cm})$ en densidades entre 1.96 y $11.05 \mathrm{ind} / \mathrm{m}^{2}$. En las lagunas El Guanal, El Cometa, el Viento, Los Ídolos y Larga entre 28.1 y $29.1^{\circ} \mathrm{C}$ y de 0.08 a 2.76 ups.

Observaciones. Especie distribuida en climas tropicales y subtropicales desde el nivel del mar hasta los $1000 \mathrm{~m}$ de altitud, en vegetación acuática sumergida, troncos hundidos, entre las raíces de la vegetación riparia, bajo rocas y en los rápidos de los ríos (Molinero, 1996; Florido et al., 2000; Álvarez et al., 2005).

Macrobrachium olfersii (Wiegmann, 1836)

Distribución. Desde cabo Fear en Carolina del Norte hasta Santa Catarina, Brasil (Holthuis, 1952; Williams, 1984; Almeida et al., 2008).

Registro en México. Vertiente occidental desde Nayarit hasta Chiapas y en la planicie costera del golfo de México desde el río Limón en Tamaulipas, Los Tuxtlas, Veracruz hasta la laguna San Pedrito en Pantanos de Centla, Tabasco (Nates y Villalobos-Hiriart, 1990; Rodríguez-Almaraz y Campos, 1996; Álvarez et al., 1999; Florido et al., 2000; Álvarez et al., 2005).

Distribución local -1 ejemplar. LT $1.74 \mathrm{~cm}$, en una densidad de $5.52 \mathrm{ind} / \mathrm{m}^{2}$. En la laguna El Guanal en 28.1 ${ }^{\circ} \mathrm{C}$ y 0.19 ups.

Observaciones. Distribuido en vegetación sumergida y entre las raíces de la vegetación riparia, troncos hundidos y sustratos rocosos cercanos a la orilla y con poca corriente. Especie registrada en ecosistemas acuáticos oligohalinos (Williams, 1984; Villalobos-Hiriart et al., 1993; Florido et al., 2000; Álvarez et al., 2005).

Familia Cambaridae Hobbs, 1942

Procambarus (Austrocambarus) llamasi (Villalobos, 1954).

Distribución. Península de Yucatán, México (VillalobosFigueroa, 1983; Hobbs, 1984). Registro en México. En la localidad de Santa Rita y estación Haro, Campeche; laguna El Sauzo en Pantanos de Centla, Tabaco; península de Yucatán (Villalobos-Figueroa, 1983; Rodríguez-Serna et al., 2002; Suárez, 2002; Álvarez et al., 2005).

Distribución local -7 ejemplares. LT promedio $1 \mathrm{~cm}$ $(0.48-1.99 \mathrm{~cm})$ en densidad de $4.08 \mathrm{ind} / \mathrm{m}^{2}$. En la laguna Punteada en $28.6^{\circ} \mathrm{C}$ y 0.61 ups.

Observaciones. Distribuido bajo piedras, hojarasca, en arroyos de corriente lenta o en aguas estancadas y en riberas en donde hay plantas acuáticas (Rodríguez-Serna et al., 2002).

Familia Panopeidae Ortmann, 1893

Rhithropanopeus harrisii (Gould, 1841)

Distribución. Estuario Miramichi, New Brunswick en Canadá hasta el norte de Brasil. Costa este del Pacífico en EUA. Costas de Portugal e Italia, mar Báltico y mar Caspio (Rodríguez, 1980; Williams, 1984; Petersen, 2006).

Registro en México. Al sur de Tampico y Pueblo Viejo, Tamaulipas; sistema lagunar de Alvarado, Veracruz; río Tonalá, laguna Chifladora, río González, laguna Mecoacán y laguna San Pedrito en Pantanos de Centla, Tabasco; laguna de Términos, Campeche (Román, 1987; 
Raz-Guzman et al., 1992; Sánchez et al., 1996; Florido et al., 2000; Suárez, 2002; Domínguez et al., 2003; Álvarez et al., 2005).

Distribución local -11 ejemplares. LT promedio $0.83 \mathrm{~cm}$ $(0.54-1.29 \mathrm{~cm})$ en densidades entre 1.1 y $21.54 \mathrm{ind} / \mathrm{m}^{2}$. En las lagunas San Pedrito, Punteada, El Cometa y Narváez entre 27.8 y $30{ }^{\circ} \mathrm{C}$ y de 0.6 a 2.76 ups.

Observaciones. Distribuido en ecosistemas limnéticos y estuarinos, en arrecifes de ostiones, sobre vegetación acuática sumergida, entre las raíces de la vegetación emergente, en troncos hundidos y detrito, bajo rocas y en sustratos lodosos y arenosos (Williams, 1984; RazGuzman y Sánchez, 1996; Florido et al., 2000; Álvarez et al., 2005).

Familia Glyptograpsidae Schubart, Cuesta y Felder, 2002 Platychirograpsus spectabilis (De Man, 1896)

Distribución. Endémico del golfo de México; Florida, Veracruz y Tabasco (Schubart et al., 2002).

Registro en México. En río Chitlán, Hidalgo; arroyo de María Andrea, Puebla; ríos Tonalá, Tuxpan, de los Hules y Tecoluta, Punta Delgada, Rancho Nuevo, El Raudal, Nautla, Chiquitos, Máquinas y Tonalá, Veracruz; ríos Macuspana y González, Tabasco (Schubart et al., 2002; Álvarez et al., 2005).

Distribución local -3 ejemplares. LT promedio $1.09 \mathrm{~cm}$ $(0.59$ a $1.93 \mathrm{~cm})$ en densidad de $2.66 \mathrm{ind} / \mathrm{m}^{2}$. En la laguna El Coco en $29.5^{\circ} \mathrm{C}$ y 0.68 ups.

Observaciones. Enterrado en sustratos suaves en las orillas de los ríos, en donde las madrigueras llegan a la zona húmeda del sustrato. También habita sobre troncos hundidos, vegetación riparia y rocas (Álvarez et al., 2005).

Familia Sesarmidae Dana, 1851

Armases cinereum (Bosc, 1802)

Distribución. Desde la bahía de Chesapeake, Maryland, este y oeste de Florida, hasta Texas, EUA (Powers, 1977; Williams, 1984; Abele, 1992).

Registro en México. Desde Tamaulipas, laguna Yucateco y ríos Tonalá y González, en Tabasco hasta Campeche (Raz-Guzman y Sánchez, 1996; Álvarez et al., 2005).

Distribución local -1 ejemplar. LT $1.07 \mathrm{~cm}$, en una densidad de $0.88 \mathrm{ind} / \mathrm{m}^{2}$. En la laguna El Coco en $29.5^{\circ} \mathrm{C}$ y 0.68 ups.

Observaciones. Distribuido en estuarios, marismas, manglares, playas, muelles, bajo troncos y raíces, en madrigueras, sustratos lodosos y arenosos. Desde la zona supralitoral hasta $50 \mathrm{~m}$ tierra adentro (Powers, 1977; Williams, 1984; Abele, 1992; Álvarez et al., 2005).

Famila Grapsidae MacLeay, 1838
Goniopsis cruentata (Latreille, 1802)

Distribución. Desde Bermuda y Florida, EUA hasta Santa Catarina, Brasil. New Croix, República Dominicana, Barbados, isla de Providencia, Curacao e islas Los Roques en las Antillas. Desde Senegal hasta el norte de Angola en África (Powers, 1977; Rodríguez, 1980; Spivak, 1997).

Registro en México. En Matamoros, Tampico, La Pesca y río Soto la Marina, Tamaulipas; Villa Cuauhtémoc, laguna Pueblo Viejo, Congregación Anáhuac, laguna de Tamiahua, laguna Tampamachoco, barra de Corazones, Tuxpan, isla Lobos, Tecolutla, estero San Agustín y sistema lagunar de Alvarado, Veracruz; laguna Yucateco y río Tonalá, Tabasco; laguna Paila y bahía de la Ascensión, Quintana Roo (Raz-Guzman et al., 1992; Álvarez et al., 2005).

Distribución local - 2 ejemplares. LT $0.85-0.87 \mathrm{~cm}$, en densidades entre 0.88 a $2.74 \mathrm{ind} / \mathrm{m}^{2}$. En la laguna El Coco en $29.5^{\circ} \mathrm{C}$ y 0.68 ups.

Observaciones. Especie asociada con la vegetación marginal en estuarios, sobre raíces y troncos de mangles, en playas lodosas y en zonas intermareales y supralitorales (Powers, 1977; Álvarez et al., 2005).

\section{Discusión}

Las 13 especies de crustáceos recolectadas en este estudio representaron el $17 \%$ de la carcinofauna registrada en los ecosistemas limnéticos del estado de Tabasco (Álvarez et al., 2005). En estudios previos realizados en Pantanos de Centla se han listado 9 especies en troncos hundidos y 8 asociadas con macrófitas sumergidas enraizadas (Florido et al., 2000; Suárez, 2002). En los 3 estudios mencionados con anterioridad coincidieron 12 especies. Este número resultó mayor que los registros para lagunas, ríos, ojos de agua, drenes, humedales y zonas de inundación en Nayarit, Nuevo León, Tamaulipas, San Luis Potosí, Veracruz, Campeche, Yucatán y Quintana Roo, que en conjunto inventariaron 10 especies de crustáceos decápodos (Rodríguez-Almaraz y Campos, 1996; Villalobos-Hiriart y Álvarez, 1999; Rodríguez-Serna et al., 2002; Martínez-Mayén y Román-Contreras, 2005).

El anfípodo Hyalella azteca fue la especie dominante (84 \% del total), está ampliamente distribuido y con frecuencia domina en ecosistemas limnéticos desde la costa Atlántica de Canadá hasta Argentina y Chile (Hann, 1995; Alcocer et al., 2000; Braccia y Batzer, 2001; Barba y Sánchez, 2007; Sánchez et al., 2007a; Sánchez et al., 2007b). Hyalella azteca se ha capturado principalmente en hábitats estructurados (Vallisneria americana (Michx.), vegetación enraizada emergente y vegetación libre flotante) $\mathrm{y}$ en menor abundancia en sustratos suaves someros sin 
vegetación (Velasco, 2004), lo que coincidió con resultados publicados para otros ecosistemas en América (Hann, 1995; Benke et al., 1999; Taylor et al., 1999; Wissinger y Gallagher, 1999; Yozzo y Diaz, 1999; Alcocer et al., 2000; Angradi et al., 2001; Cheruvelil et al., 2002).

La extensa distribución geográfica de Hyalella azteca, Macrobrachium acanthurus y $M$. olfersii se ha explicado en función de su tolerancia a las variaciones de salinidad, temperatura y concentraciones de oxígeno disuelto (Taylor et al., 1999, Alcocer et al. 2000; Álvarez et al., 2005). En el caso de Potimirim mexicana, su amplia distribución se ha asociado con su tolerancia a condiciones ambientales adversas, como contaminación por hidrocarburos y aguas negras de origen urbano (Álvarez et al., 2005), lo que también se aplica para $H$. azteca, ya que ha sido registrada en casi total ausencia de oxígeno disuelto, altas temperaturas y pH alcalino (Taylor et al., 1999, Álvarez et al., 2005).

La distribución de Balanus improvisus, Uhlorchestia ulheri, Platychirograpsus spectabilis, Armases cinereum y Goniopsis cruentata estuvo restringida a la zona de influencia marina de Pantanos de Centla, que se localiza en la subcuenca de Tres Brazos, y su extensión varía drásticamente por los pulsos de inundación (Sánchez et al., 2007a; Salcedo et al., 2009). El cirripedio B. improvisus se ha registrado en áreas polihalinas y marinas (Britton y Morton, 1988; Florido et al., 2000). En cambio, la distribución espacial restringida de las otras 4 especies (U. uhleri, A. cinereum, G. cruentata y P. spectabilis) en Pantanos de Centla no se puede vincular con su tolerancia a la salinidad, ya que son consideradas con distribución amplia en relación con la salinidad y asociación con el hábitat (Bousfield, 1986; Raz-Guzman y Sánchez, 1996; Álvarez et al., 2005; Velasco et al., 2005).

En éste y otro estudio de la misma área publicado previamente (Florido et al., 2000), la distribución del cirripedio Balanus improvisus y del isópodo Sphaeroma terebrans estuvo restringida a los troncos hundidos. Aunque ambas especies han sido recolectadas en las raíces de $R$. mangle en otros ecosistemas (Britton y Morton, 1988; Kensley y Schotte, 1989; Álvarez et al., 2005), en Pantanos de Centla esta especie de mangle está limitada a la subcuenca de Tres Brazos (Sánchez et al., 2007a), que es donde se distribuyó $B$. improvisus; en cambio, la distribución de $S$. terebrans fue más amplia, por no estar restringida a condiciones estuarinas. Por tanto, el único hábitat para S. terebrans y otros invertebrados acuáticos sésiles, taladradores y xilófagos en Pantanos de Centla son los troncos hundidos (Britton y Morton, 1988; Johnson y Kennedy, 2003; Gulis et al., 2004; Hernández et al., 2005; Montalvo-Urgel, datos no publicados).

Los troncos hundidos son un hábitat alternativo para las restantes 11 especies de crustáceos listados, pues su distribución es independiente de la presencia de sustratos duros. Entre estas 11 especies, Hyalella azteca, Uhlorchestia uhleri, Potimirim mexicana, Macrobrachium acanthurus, M. hobbsi, Procambarus (Austrocambarus) llamasi y Rhithropanopeus harrisii fueron previamente capturadas en otros hábitats en Pantanos de Centla. En otros ecosistemas, la distribución de estas 7 especies así como de Macrobrachium olfersii y Armases cinereum está relacionada con hábitats estructurados y cavidades en el sustrato (Williams, 1984; Bousfield y Heard, 1986; Abele, 1992; Villalobos-Hiriart et al., 1993; Raz-Guzman y Sánchez, 1996; Corona et al., 2000; Angradi et al., 2001; Cheruvelil et al., 2002; Rodríguez-Serna et al., 2002; Álvarez et al., 2005; Velasco et al., 2005; Barba y Sánchez, 2007), los cuales proporcionan una complejidad física del hábitat similar a la de los troncos hundidos.

El camarón Potimirim mexicana, el cangrejo del lodo Rhithropanopeus harrisii junto con el isópodo taladrador Sphaeroma terebrans representaron las especies detritívoras que se alimentan de la materia orgánica acumulada en las galerías de oquedades de los troncos hundidos. El átido $P$. mexicana está considerado un importante reciclador de detrito (Crowl et al., 2001); el cangrejo del lodo consume principalmente detrito, anfípodos y copépodos (Britton y Morton, 1988), y el isópodo se ha registrado como especie que se alimenta de las bacterias y hongos establecidos en galerías que él construye (Britton y Morton, 1988; Ellison y Farnsworth, 1990). Las galerías proveen refugio contra la depredación; sobre todo en los troncos, donde estos microhábitats se han incrementado en relación con el tiempo de permanencia del tronco en el agua y el grado de descomposición del mismo (Lehane et al., 2002; Tietje et al., 2002; Brooks et al., 2004; Heinemann y Kitzberger, 2006). Las galerías en los troncos hundidos son utilizadas por Procambarus (Austrocambarus) llamasi, cuya amplia distribución en el sureste de México (Villalobos-Figueroa, 1983; Rodríguez-Serna et al., 2002; Álvarez et al., 2005) se relaciona con la presencia de tipos de sustrato y de vegetación que facilitan la construcción de refugios contra depredadores y la acumulación de detrito (RodríguezSerna et al., 2002). Los acociles del género Procambarus depredan especies suaves y de baja movilidad (Nyström et al., 1996) que se han recolectado en los troncos hundidos en Pantanos de Centla (Montalvo-Urgel, datos no publicados).

En los humedales tropicales, la vegetación marginal y la sumergida enraizada se consideran sitios de alimentación, reproducción y protección, ya que son hábitats de elevada heterogeneidad y complejidad estructural (Pelicice y Agostinho, 2006; Rozas y Minello, 2006; Barba y Sánchez, 2007; Cetra y Petrere, 2007; Genkai-Kato, 2007), 
al igual que los troncos hundidos (Everett y Ruiz, 1993). Sin embargo, la abundancia y el número de especies de crustáceos en los troncos hundidos resultaron menores en este estudio que lo registrado en los hábitats con vegetación (Sánchez et al., 2007a).

En Pantanos de Centla, los troncos hundidos representan tanto el principal sustrato duro disponible para especies sésiles y taladradoras como un hábitat alternativo para la fauna asociada. Así, a pesar del área mínima que cubren en los ecosistemas acuáticos permanentes en los Pantanos de Centla, que es menor al $0.01 \%$ de su extensión (Sánchez et al., 2007a), resalta la importancia de incluirlos en los programas de manejo de este ecosistema.

\section{Literatura citada}

Abele, L.G. 1992. A review of the grapsid crab genus Sesarma (Crustacea: Decapoda: Grapsidae) in America, with the description of a new genus. Smithsonian Contributions to Zoology 527:1-60.

Abele, L. G. y W. Kim. 1986. An illustrated guide to the marine decapod crustaceans of Florida. Department of Environmental Regulation Technical Series 8:1-213.

Alcocer, J., E. Escobar-Briones, L. Peralta y F. Álvarez. 2000. Population structure of the macrobenthic amphipod Hyalella azteca Saussure (Crustacea: Peracarida) on the littoral zone of six crater lakes. In Modern approaches to the study of Crustacea, E. Escobar-Briones y F. Álvarez (eds.). Kluwer Academic/Plenum, New York. 355 p.

Almeida, A. O., P. A. Coelho, J. R. Luz, J. T. A. Santos y N. F. Ferraz. 2008. Decapod crustaceans in fresh waters of southeastern Bahia, Brazil. Revista de Biología Tropical 56:1225-1254.

Álvarez, F., J. L. Villalobos-Hiriart y R. Robles. 1999. Listas y comentarios sobre los crustáceos decápodos de Veracruz, México. Anales del Instituto de Biología, Universidad Nacional Autónoma de México, Serie Zoología 70:1-27.

Álvarez, F., J.L. Villalobos-Hiriart y R. Robles. 2005. Crustáceos. In Biodiversidad del estado de Tabasco, Cap. 8, J. Bueno, F. Álvarez y S. Santiago (eds.). Instituto de Biología, UNAM/ Comisión Nacional para el Conocimiento y Uso de la Biodiversidad, México, D.F. p. 177-194.

Angradi, T. R., S. M. Hagan y K. W. Abele. 2001. Vegetation type and the intertidal macroinvertebrate fauna of a brackish marsh: Phragmites vs. Spartina. Wetlands 21:75- 92.

Bacon, P. R. 1996. Wetlands and biodiversity. In Wetlands, biodiversity and the Ramsar convention: the role of the convention on wetlands in the conservation and wise use of biodiversity, A. J. Hails (ed.). Ramsar Convention Bureau, Gland. p. 17.

Barba, E. y A. J. Sánchez. 2007. A new record of Hyalella azteca Saussure, 1858 (Amphipoda, Hyalellidae) from the LermaChapala Basin, Mexican Pacific coast. Crustaceana 80:625630.

Barnard, J. L. y G. S. Karaman. 1991. The families and genera of marine gammaridean Amphipoda (except marine gammaroids). Part 1 and 2. Records of the Australian Museum, Supplement 13:1-866.

Bautista-Jiménez, A., A. García-Muñiz, P. Pérez-Alejandro y J. C. Romero-Gil. 2000. Caracterización de la Reserva de la Biosfera Pantanos de Centla. Universidad y Ciencia 15:1528.

Benke, A. C., G. M. Ward y T. D. Richardson. 1999. Beaverimpounded wetlands of the southeastern coastal plain. Habitat-specific composition and dynamics of invertebrates. In Invertebrates in freshwather wetlands of North America, D. P. Batzer, R. B. Rader y S. A. Wissinger (eds.). Ecology and management. Wiley, Toronto, Ontario. p. 217- 245.

Bousfield, E. L. 1973. Shallow-water gammaridean Amphipoda of New England. Cornell University Press, Ithaca, New York. 312 p.

Bousfield, E. L. y R. W. Heard. 1986. Systematics, distributional ecology, and some host-parasite relationships of Uhlorchestia uhleri (Shoemaker) and U. spartinophila, new species (Crustacea: Amphipoda), endemic to salt marshes of the Atlantic coast of north America. Journal of Crustacean Biology 6:264- 274.

Braccia, A. y D. P. Batzer. 2001. Invertebrates associated with woody debris in a southeastern U.S.A. forested floodplain wetland. Wetlands 21:18-31.

Britton, J. C. y B. Morton. 1988. Shore ecology of the Gulf of Mexico. University of Texas Press, Austin. 387 p.

Brooks, A. P., P. C. Gehrke, J. D. Jansen y T. B. Abbe. 2004. Experimental reintroductions of woody debris on the Williams river, NSW: geomorphic and ecological responses. River Research and Applications 20:513- 536.

Celis, A. 2004. Taxonomía y patrones de distribución de los cirripedios (Crustacea: Cirripedia: Thoracica) sublitorales de la parte sur del golfo de México. Tesis, Maestría Posgrado en Ciencias Biológicas, Universidad Nacional Autónoma de México, México, D.F. 152 p.

Cetra, M. y J. R. M. Petrere. 2007. Associations between fish assemblages and riparian vegetation in the Corumbataí River Basin (SP). Brazil Journal Biology 67:191-195.

Collier, K. J. y J. N. Halliday. 2000. Macroinvertebrate wood associations during decay of plantation pine in New Zealand pumice-bed streams: stable habitat of trophic subsidy. Journal of the North American Benthological Society 19:94-111.

Corona, A., L. A. Soto y A. J. Sánchez. 2000. Epibenthic amphipod abundance and pink shrimp Farfantepenaeus duorarum predation efficiency in habitats with differential physical complexity. Journal of Experimental Marine Biology and Ecology 253:33-48.

Corona, A. y A. Raz-Guzman. 2003. Distribución geográfica de los anfípodos e isópodos (Crustacea: Peracarida: Amphipoda e Isopoda) de los sistemas estuarinos de Michoacán, México. In Contribuciones al estudio de los crustáceos del Pacífico este 2, M. Hendrickx (ed.). Instituto de Ciencias del Mar y Limnología, UNAM, México, D.F. p. 219- 225.

Crowl, T. A., W. H. McDowell, A. P. Covich y S. L. Johnson. 2001. Freshwater shrimp effects on detrital processing and nutrients in a tropical headwater stream. Ecology 82:775-783. 
Cruz-Ascencio, M., R. Florido, E. Barba y A. J. Sánchez. 2009. Colonización de carcinofauna epibentónica en estructuras artificiales de Vallisneria americana en un humedal tropical mesoamericano. Memorias del Primer Simposium para el Conocimiento de los Recursos Costeros del Sureste de México, Universidad Autónoma del Carmen, Ciudad del Carmen, Campeche, 3-5 de junio. p. 184-185.

Cummins, K. W., J. R. Sedell, F. J. Swanson, G. W. Minshall, S. G. Fisher, C. E. Cushing, R. C. Petersen y R. L. Vannote. 1983. Organic matter budgets for stream ecosystems: problems in their evaluation. In Stream ecology, application and testing of general ecological theory plenum, J. R. Barnes y G. W. Minshall (eds.). New York. p. 299-353.

Chace, F. A. y H. H. Hobbs, Jr. 1969. The freshwater and terrestrial decapod crustaceans of the West Indies with special reference to Dominica. United States National Museum Bulletin 292:1-257.

Chace, F. A. 1972. The shrimps of the Smithsonian-Bredin Caribbean expeditions with a summary of the West Indian shallow-water species (Crustacea: Decapoda: Natantia). Smithsonian Institution Press, Washington, D.C. 141 p.

Cheruvelil, K. S., P. A. Soranno, J. D. Madsen y M. J. Roberson. 2002. Plant architecture and epiphytic macroinvertebrate communities: The role of an exotic dissected macrophyte. Journal of the North American Benthological Society 21:261-277.

Domínguez, J. C., A. J. Sánchez, R. Florido y E. Barba. 2003. Distribución de macrocrustáceos en Laguna Mecoacán, al sur del golfo de México. Hidrobiológica 13:127-136.

Ellison, A. M. y E. J. Farnsworth. 1990. The ecology of Belizean mangrove-root fouling communities. I. Epibenthic fauna are barriers to isopod attack of red mangrove roots. Journal of Experimental Marine Biology and Ecology 142:91-104.

Everett, R. A. y G. M. Ruiz. 1993. Coarse woody debris as a refuge from predation in aquatic communities. Oecologia 93:475-486.

Fausch, K. D. y T. G. Northcote. 1992. Large woody debris and salmonid habitat in a small coastal British Columbia Stream. Canadian Journal of Fisheries and Aquatic Sciences 49:682-693.

Florido, R., A. J. Sánchez y J. L. Villalobos-Hiriart. 2000. Macrocrustáceos asociados con troncos hundidos en la laguna San Pedrito, Reserva de la Biosfera Pantanos de Centla. Universidad y Ciencia 15:115-128.

Genkai-Kato, M. 2007. Macrophyte refuges, prey behaviour and trophic interactions: consequences for lake water clarity. Ecology Letters 10:105-114.

González, E.R. y L. Watling. 2001. Three new species of Hyalella from Chile (Crustacea: Amphipoda: Hyalellidae). Hydrobiologia 464: 175- 199.

González, E. R. y L. Watling. 2002. Redescription of Hyalella azteca from its type locality, Veracruz, México (Amphipoda: Hyalellidae). Journal of Crustacean Biology 22:173-183.

Granados, A. 1984. Aspectos reproductivos del "camarón prieto" (Wiegman, 1836) en la cuenca del río González, Tabasco, México (Crustacea: Decapoda: Palaemonidae). Anales del Instituto de Ciencias del Mar y Limnología, Universidad Nacional Autónoma de México 11:1-22.
Guevara, E., A. J. Sánchez, C. Rosas, M. Mascaró y R. Brito. 2007. Asociación trófica de peces distribuidos en vegetación acuática sumergida en laguna de Términos, sur del golfo de México. Universidad y Ciencia 23:151-166.

Gulis, V., A. D. Rosemond, K. Suberkropp, H. Weyers y J. P. Benstead. 2004. Effects of nutrient enrichment on the decomposition of wood and associated microbial activity in streams. Freshwater Biology 49:1437-1447.

Hann, B. J. 1995. Invertebrate associations with submerged aquatic plants in a prairie wetland. UFS (Delta Marsh) Annual Report 30:78-84.

Harmon, M. E., J. F. Franklin, F. J. Swanson, P. Sollins, S. V. Gregory, J. D. Lattin, N. H. Anderson, S. P. Clien, N. G. Aumen, J. R. Sedell, G. W. Lienkaemper, K. Cromack y K. W. Cummins. 1986. Ecology of coarse woody debris in temperate ecosystems. In Advances in ecological research 15, A. E. MacFadyen y D. Ford (eds.). Academic, Orlando, Florida. 436 p.

Heck, K. L. y L. B. Crowder. 1991. Habitat structure and predatorprey interactions in vegetated aquatic systems. In Habitat structure: the physical arrangement of objects in space, S. S. Bell, E. D. McCoy y H. R. Mushinsky (eds.). Chapman and Hall, London. p. 281- 299.

Heinemann, K. y T. Kitzberger. 2006. Effects of position, understorey vegetation and coarse woody debris on tree regeneration in two environmentally contrasting forests of north-western Patagonia: a manipulative approach. Journal of Biogeography 33:1357-1367.

Hernández, O., R. W. Merritt y M. S. Wipfli. 2005. Benthic invertebrate community structure is influenced by forest succession after clearcut logging in southeastern Alaska. Hydrobiologia 533:45-59.

Hobbs, H. H., Jr. 1984. On the distribution of the crayfish genus Procambarus (Decapoda:Cambaridae). Journal of Crustacean Biology 4:12-24.

Holthuis, L. B. 1952. A general revision of the Palaemonidae. Allan Hancock Foundation Occasional Papers 12:1-396.

Johnson, Z. B. y J. H. Kennedy. 2003. Macroinvertebrate assemblages of submerged woody debris in the Elm Fork of the Trinity River, Texas. Journal of Freshwather Ecology 18:187-197.

Kensley, B. y M. Schotte. 1989. Guide to the marine isopod crustaceans of the Caribbean. Smithsonian Institution Press, Washington, D.C., 308 p.

Lehane, B. M., P. S. Giller, J. O. Halloran, C. Smith y J. Murphy. 2002. Experimental provision of large woody debris in streams as a trout management technique. Aquatic Conservation: Marine and Freshwater Ecosystems 12:289-311.

Lévêque, C., E. V. Balian y K. Martens. 2005. An assessment of animal species diversity in continental waters. Hydrobiologia 542:39-67.

Lincoln, R. J. 1979. British marine Amphipoda: Gammaridea. British Museum (Natural History), London. 658 p.

Lippson, A. J. y R. L. Lippson. 1984. Life in the Chesapeake Bay. Johns Hopkins University Press, Baltimore, Maryland. $230 \mathrm{p}$.

Lockaby, B. G., B. D. Keeland, J. A. Stanturf, M. D. Rice, G. 
Hodges y R. M. Governo. 2002. Arthropods in decomposing wood of the Atchafalaya River Basin. Southeastern Naturalist 1:339-352.

Martin, J. W. y G. E. Davis. 2001. An updated classification of the recent Crustacea. Natural History Museum of Los Angeles County, Science Series 39:1-124.

Martínez-Mayén, M. y R. Román-Contreras. 2005. Notes on the fertility of Potimirim mexicana (de Saussure, 1857) (Decapoda, Atyidae) in Laguna de Términos, Gulf of Mexico. Crustaceana 78:29-40.

Myers, A. A. y J. K. Lowry. 2003. A phylogeny and a new classification of the Corophiidea (Amphipoda). Journal of Crustacean Biology 23:443-485.

Mejía-Ortíz, L. M., F. Álvarez, R. Román y J. A. Viccon-Pale. 2001. Fecundity and distribution of freshwater prawns of the genus Macrobrachium in the Huitzilapan river, Veracruz, Mexico. Crustaceana 74:69-77.

Molinero, J. C. 1996. Biología del langostino Macrobrachium hobbsi (Nates y Villalobos-Hiriart, 1990) de la región de Los Tuxtlas, Veracruz. Tesis, Facultad de Ciencias, Universidad Nacional Autónoma de México, México, D.F. 54 p.

Nates, J. C. y J. L. Villalobos-Hiriart. 1990. Dos nuevas especies de camarones de agua dulce del género Macrobrachium Bate (Crustacea, Decapoda, Palaemonidae), de la vertiente occidental de México. Anales del Instituto de Biología, Universidad Nacional Autónoma de México, Serie Zoología 61:1-11.

Nyström, P., C. Brönmark y W. Granéli. 1996. Patterns in benthic food webs: a role for omnivorous crayfish? Freshwater Biology 36:631-646.

O'Connor, N. A. 1991. The effects of habitat complexity on the macroinvertebrates colonizing wood substrates in lowland streams. Oecologia 85:504-512.

Pelicice, F. M. y A. A. Agostinho. 2006. Feeding ecology of fishes associated with Egeria spp patches in a tropical reservoir. Brazilian Ecology Freshwater Fish 15:10-19.

Pilsbry, H. A. 1916. The sessile bamacles (Cirripedia) contained in the collections of the U.S. National Museum, including a monograph of the American species. United States National Museum Bulletin, Smithsonian Institution, Washington, D.C. $413 \mathrm{p}$.

Pennak, R. M. 1978. Freshwater invertebrates of the United States. Wiley-Interscience, New York. 803 p.

Petersen, C. 2006. Range expansion in the northeast Pacific by an estuary mud crab - a molecular study. Biological Invasions 8:565-576.

Powers, L. W. 1977. A catalogue and bibliography to the crabs (Brachyura) of the Gulf of México. Contribution of Marine Science Supplement 20:1-190.

Primavera, J. H. 1997. Fish predation on mangrove-asociated penaeids. The role of structures and substrate. Journal of Experimental Marine Biology and Ecology 215:205-216.

Raz-Guzman, A. y M. Hermoso. 2002. Distribución espacial y afinidad zoogeográfica de los camarones carideos y cangrejos anomuros y braquiuros de los sistemas litorales de Michoacán. In Contribuciones al estudio de los crustáceos del Pacífico este, M. E. Hendrickx (ed.). Instituto de Ciencias del Mar y Limnología, UNAM, México, D.F. p. 265- 278.
Raz-Guzman, A. y A. J. Sánchez. 1996. Catálogo ilustrado de cangrejos braquiuros (Crustacea) de la Laguna de Tamiahua, Veracruz, México. Cuadernos 31. Instituto de Biología, UNAM, México, D.F. 51 p.

Raz-Guzman, A., A. J. Sánchez y L. A. Soto. 1992. Catálogo ilustrado de cangrejos braquiuros y anomuros (Crustacea) de la Laguna de Alvarado, Veracruz,

México. Cuadernos 14. Instituto de Biología, UNAM, México, D.F. 51 p.

Rocha-Ramírez, A., A. Ramírez, R. Chávez y J. Alcocer. 2007. Invertebrate assemblages associated with root masses of Eichhornia crassipes (Mart.) Solms-Laubach 1883 in the Alvarado Lagoon System, Veracruz, Mexico. Aquatic Ecology 41:319-333.

Rodríguez, B. 1993. Shrimps. In FAO species identification sheets for fishery purposes: Field guide to the commercial marine and brackish-water resources of the northern coast of south America, F. Cervigon, R. Cipriani, W. Fischer, L. Caribaldi, M. Hendrickx, A. J. Lemus, R. Márquez, J. M. Poutiers, G. Robaina y B. Rodríguez (eds.). Virginia. p. 281- 299

Rodríguez, G. 1980. Los crustáceos decápodos de Venezuela. Instituto Venezolano de Investigaciones Científicas, Caracas, 494 p.

Rodríguez-Almaraz, G. y E. Campos. 1996. New locality records of freshwater decapods from México (Crustacea: Atyidae, Cambaridae and Palaemonidae). Proceedings of the Biological Society of Washington 109:34-38.

Rodríguez-Serna, M., C. Carmona-Osalde, J. L. ArredondoFigueroa y M. A. Olvera-Novoa. 2002. Distribución geográfica actual de Procambarus (Austrocambarus) llamasi (Cambaridae) en la Península de Yucatán. Hidrobiológica 12:1-5.

Román, R. 1987. Características ecológicas de los crustáceos decápodos de la laguna de Términos. In Ecología de los ecosistemas costeros en el sur del golfo de México: la región de la Laguna de Términos, A. Yáñez- Arancibia y J. W. Day (eds.). Instituto de Ciencias del Mar y Limnología, UNAM, México, D.F./Coastal Ecological Institute, LSU, Baton Rouge, Louisiana p. 233- 245.

Rozas, L. P. y T. J. Minello. 2006. Nekton use Vallisneria americana Michx. (wild celery) beds and adjacent habitats in coastal Louisiana. Estuaries and Coasts 29:297-310.

Salcedo, M. A., G. de la Lanza y A. J. Sánchez. 2009. Efectos de los pulsos de inundación en la fisicoquímica del agua en Pantanos de Centla. Primer Simposium para el Conocimiento de los Recursos Costeros del Sureste de México, Universidad Autónoma del Carmen, Ciudad del Carmen, Campeche, 3-5 de junio. p. 71-72.

Sammons, S. M. y M. J. Maceina. 2006. An evaluation of different structures to age freshwater fish from a northeastern US river. Fisheries Management and Ecology 13:237-246.

Sánchez, A. J., A. Raz-Guzman y E. Barba. 1996. Habitat value of seagrasses for decapods in tropical coastal lagoons of the southwestern Gulf of Mexico. In Seagrasses biology: Proceedings of an international workshop, J. R. Kuo, C. Phillips, D. I. Walker y H. Kirkman (eds.). Rottnest Island, January, 25-29. University of Western Australia. 
p. 233-240.

Sánchez, A. J., M. A. Salcedo, R. Florido, A. Armenta, C. Rodríguez, A. Galindo y E. Moguel. 2007a. Pantanos de Centla, un humedal costero tropical. In Las aguas interiores de México, conceptos y casos, G. de La Lanza y S. Hernández (eds.). AGT, México, D.F. . p. 401-420.

Sánchez, M. R., M. G. Oliva, J. M. Castillo y A. Lugo. 2007b. Lago urbano del parque Tezozómoc, Distrito Federal. In Las aguas interiores de México, conceptos y casos, G. de la Lanza y S. Hernández (eds.). AGT, México, D.F. p.127-139.

Saussure, H. 1858. Mémoire sur divers crustacés nouveaux de Antilles et du Mexique. Memoire de la Societe Physique du Histoire Naturrelle 14:417-496.

Schubart, C. D., J. A. Cuesta y D. L. Felder. 2002. Glyptograpsidae, a new branchyuran family from Central America: larval and adult morphology, and molecular phylogeny of the Grapsoidea. Journal of Crustacean Biology 22:28-44.

Schultz, G. A. 1969. How to know the marine isopod crustaceans. Brown and Dubuque, Iowa. 359 p.

Sedell, J. R., F. H. Everest y F. J. Swanson. 1982. Fish habitat and streamside management: past and present. Proceedings of the Society of American Foresters. Annual Meeting. Bethesda. p. 244-245.

Serejo, C. S. 2004. Cladistic revision of talitroidean amphipods (Crustacea, Gammaridea), with a proposal of a new classification. Zoologica Scripta 33:551-586.

Spivak, E. D. 1997. Cangrejos estuariales del Atlántico sudoccidental $\left(25^{\circ}-45^{\circ} \mathrm{S}\right)$ (Crustacea: Decapoda: Brachyura). Investigación Marina 25:105-120.

Suárez, J. A. 2002. Distribución de macrocrustáceos decápodos asociados a dos hábitats contrastantes en los Pantanos de Centla, Tabasco. Tesis, Universidad Juárez Autónoma de Tabasco, Villahermosa. 39 p.

Taylor, B. E., D. A. Leeper, M. A. McClure y A. E. De Biase. 1999. Carolina Bay. Ecology of aquatic invertebrates and perspectives on conservation. In Invertebrates in freshwater wetlands of North America: Ecology and Management, D. P. Batzer, R. B. Rader y S. A. Wissinger (eds.). Wiley, Hoboken, New Jersey. p.167-196.

Thorp, J. H., E. M. McEwan, M. F. Flynn y F. R. Haver. 1985. Invertebrate colonization of sumerged wood in a cypresstupelo swamp and blackwater stream. Ecology 66:14911503.

Tietje, W. D., K. L. Waddell, J. K. Vreeland y C. L. Bolsinger. 2002. Coarse woody debris in oak woodlands of California. Society of American Foresters 17:139-146.

Triska, F. J., J. R. Sedell y S. V. Gregory. 1982. Coniferous forest streams. In Analysis of coniferous forest ecosystems in the western United States, R. L. Edmonds (ed.). United States International Biological Program Synthesis Series 14. Hutchinson Ross, Stroudsburg, Pennsylvania. p. 292-332.

Vargas, R. y J. Cortés. 1999. Biodiversidad marina de Costa Rica (Crustacea: Decapoda: Penaeidae, Sergestoidea,
Stenopodidea, Caridea, Thalassinidea, Palinura). Revista de Biología Tropical 47:877-885.

Velasco, C. 2004. Distribución espacial y temporal de anfípodos (Peracarida: Gammaridea) en la Reserva de la Biosfera Pantanos de Centla. Tesis, División Académica de Ciencias Biológicas, Universidad Juárez Autónoma de Tabasco, Villahermosa. 28 p.

Velasco, C., A. J. Sánchez y R. Florido. 2005. New locality record of Uhlorchestia uhleri (Peracarida: Amphipoda: Talitridae) in the coastal zone of the southern Gulf of Mexico. Crustaceana 78:499-502.

Velázquez-Villegas, G. 1994. Los recursos hidrológicos del estado de Tabasco. Ensayo monográfico. Universidad Juárez Autónoma de Tabasco, Villahermosa. 242 p.

Villalobos-Figueroa, A. 1959. Contribución al conocimiento de los Atyidae de México II (Crustacea, Decapoda). Estudio de algunas especies del género Potimirim (Ortmannia), con descripción de una especie nueva en Brasil. Anales del Instituto de Biología, Universidad Nacional Autónoma de México 30:269-330.

Villalobos-Figueroa, A. 1982. Decapoda. In Aquatic biota of México, Central America and the West Indies, H. Hurlbert y A. Villalobos-Figueroa (eds.). San Diego State University Foundation, California. p. 215-239.

Villalobos-Figueroa, A. 1983. Crayfishes of Mexico (Crustacea: Decapoda). Smithsonian Institution Press, Washington, D.C. $276 \mathrm{p}$.

Villalobos-Hiriart, J. L. y F. Álvarez. 1999. A new species of Macrobrachium (Crustacea: Decapoda: Palaemonidae), with abbreviated development, from Veracruz, México. Proceedings of the Biological Society of Washington 112:746-753.

Villalobos-Hiriart, J. L., A. Díaz-Barriga y E. Lira-Fernández. 1993. Los crustáceos de agua dulce de México. Revista de la Sociedad Mexicana de Historia Natural 44:267-290.

Williams, A. B. 1984. Shrimps, lobsters and crabs of the Atlantic coast of the Eastern United States, Maine to Florida. Smithsonian Institution Press, Washington, D.C. 550 p.

Wissinger, S. A. y L. J. Gallagher. 1999. Beaver pond wetlands in northwestern Pennsylvania. Modes of colonization and succession after drought. In Invertebrates in freshwater wetlands of North America: Ecology and management, D. P. Batzer, R. B. Rader y S. A. Wissinger (eds.). Wiley, Hoboken, New Jersey. p. 333-362.

Yamazaki, Y., S. Haramoto y T. Fukasawa. 2006. Habitat uses of freshwater fishes on the scale of reach system provided in small streams. Environmental Biology of Fishes 75:333341.

Yozzo, D. J. y R. J. Diaz. 1999. Tidal freshwater wetlands. Invertebrate diversity, ecology, and functional significance. In Invertebrates in freshwater wetlands of North America: ecology and management, D. P. Batzer, R. B. Rader y S. A. Wissinger (eds.). Wiley, Hoboken, New Jersey. p. 889-918. 